\title{
Raman fiber lasers with a random distributed feedback based on Rayleigh scattering
}

\author{
D. V. Churkin, ${ }^{1, *}$ S. A. Babin, ${ }^{1}$ A. E. El-Taher, ${ }^{2}$ P. Harper, ${ }^{2}$ S. I. Kablukov, ${ }^{1}$ V. Karalekas, ${ }^{2}$ J. D. Ania-Castañón, ${ }^{3}$ \\ E. V. Podivilov, ${ }^{1}$ and S. K. Turitsyn ${ }^{2}$ \\ ${ }^{1}$ Institute of Automation and Electrometry, SB RAS, Novosibirsk 630090, Russia \\ ${ }^{2}$ Photonics Research Group, Aston University, Birmingham, United Kingdom \\ ${ }^{3}$ Instituto de Óptica, CSIC, E-28006 Madrid, Spain \\ (Received 9 June 2010; published 22 September 2010)
}

\begin{abstract}
We demonstrate lasing based on a random distributed feedback due to the Raman amplified Rayleigh backscattering in different types of cavities with and without conventional point-action reflectors. Quasistationary generation of a narrowband spectrum is achieved despite the random nature of the feedback. The generated spectrum is localized at the reflection or gain spectral maxima in schemes with and without point reflectors, respectively. The length limit for a conventional cavity and the minimal pump power required for the lasing based purely on a random distributed feedback are determined.
\end{abstract}

DOI: 10.1103/PhysRevA.82.033828

PACS number(s): 42.81.Dp, 42.55.Ye, 42.65.Dr, 42.55.Zz

\section{INTRODUCTION}

The concept of random lasers exploiting multiple scattering in amplifying disordered media to generate coherent light has attracted a great deal of attention in recent years (see review articles [1,2] and references therein). A basic laser scheme normally requires two key elements: a gain medium that provides amplification and an optical cavity that traps the light, creating a positive feedback. Lasing occurs when the total gain in the cavity overcomes the total cavity loss. Spectral and temporal characteristics of conventional lasers are determined both by the gain medium and by the cavity design that defines lasing frequencies-longitudinal modes-while transverse modes define spatial properties of the output beam. On the contrary, in random lasers with no cavity (or with an open cavity), the multiple scattering of a significant portion of the photons in an amplifying disordered medium increases the effective optical path, resulting in lasing that was theoretically predicted in [3] and experimentally demonstrated in [4-6]. The spectral, temporal, and spatial performances of the output beam in random lasers are determined by the buildup of the radiation in randomly embedded local spatial modes that may coexist with nonlocalized extended modes [7,8].

Usually, the random nature of generation of radiation provides random lasers with special features compared to conventional cavity lasers, such as pulsed operation, complex nonlocalized emission spectra, or an irregular and unstable angular dependence of the output beam [2]. Low-dimensional random systems can be used to overcome these problems. It has been shown that random multilayers with disorder may provide directional output [9]. Directional pulsed random lasing has been also demonstrated in the photonic crystal fiber with the hollow core filled with a suspension of $\mathrm{TiO}_{2}$ nanoparticles in a rhodamine $6 \mathrm{G}$ dye solution [10]. In this way, the fiber waveguide properties are combined with traditional bulk random material (amplifying dye with randomly scattering nanoparticles) providing one-dimensional random lasing.

An alternative approach is to use the intrinsic disorder in the fiber. Indeed, the refractive index of standard

\footnotetext{
*churkin@iae.nsk.su
}

telecommunication fiber has submicron-scale inhomogeneities, which are random distributed along the fiber. During propagation, the light is scattered by the inhomogenities obeying the Rayleigh law. Rayleigh scattering (RS) is responsible for setting a lower limit to losses in telecommunication fibers, with a value of about $0.2 \mathrm{~dB} / \mathrm{km}$ at wavelengths around $1.55 \mu \mathrm{m}$, corresponding to the transparency window of silica glasses [11]. To achieve random lasing in a fiber, only the fraction of the Rayleigh scattered radiation which is reflected back into the fiber waveguide, that is, backscattering, can be used. The backscattering intensity in a single-mode telecom fiber is about 600 times lower than the total scattering intensity because the fiber geometry accepts only backscattered waves in a small cone described by a numerical aperture. Hence, the Rayleigh backscattering coefficient is extremely small, having a typical value of $\varepsilon=4.5 \times 10^{-5} \mathrm{~km}^{-1}$ only [12]. This feature makes the system considered here rather different from conventional random lasers, where strong scattering effect is used.

In principle, even a weak feedback might be important for laser devices and may influence its lasing characteristics. However, in a fiber there is an additional possibility for controling the feedback level. Namely, the backscattered light can be easily amplified, thus providing a much more visible impact. Indeed, a conventional fiber pumped by a laser source exhibits a Raman gain for the light shifted to the longer wavelengths relative to that for the pump laser. In long fibers, the Raman amplified Rayleigh backscattering begins to play a significant role and, in particular, limits the maximum achievable length of the Raman fiber laser (RFL) cavity with a resolvable longitudinal mode structure to a value of $\sim 270 \mathrm{~km}$ [13]. Moreover, it was shown that in a 10-km-long Raman amplifier, irregular lasing starts at high pump power due to the distributed Rayleigh feedback only, without any point-action mirrors [14]. The generated spectrum features random spikes on top of the Raman gain profile with temporal instabilities. Similar results were obtained for Raman amplifiers in [15], where it was also noted that four-wave mixing might suppress the Rayleigh instabilities of Raman amplifiers in low-dispersion fibers. It is known that distributed $\mathrm{RS}$, together with stimulated Brillouin scattering (SBS), results in the self- $Q$-switched operation of an ytterbiumdoped fiber laser [16]. In a Brillouin laser (i.e., laser based 
on the SBS-induced gain), double-distributed RS feedback results in a significant reduction of the laser linewidth [17]. Temporally and spectrally unstable laser generation has been also obtained in 10-km-long RFL operating via cooperative SBS-RS feedback [18]. Stationary Raman lasing is feasible in a hybrid cavity formed by a fiber Bragg grating (FBG) and RS-based distributed mirror in an 11-km-long fiber [19] providing a narrow spectrum following FBG reflection bandwidth. This system was proposed for remote sensing exploiting the sensitivity of the FBG wavelength to the environment.

Finally, in a recent article [20], stationary directional laser generation with a localized narrow spectrum was achieved in a fiber cavity formed only by a random distributed feedback provided by Raman amplified Rayleigh backscattering. In the configuration discussed in [20], a standard telecommunication fiber of $83 \mathrm{~km}$ total length was pumped from its center in opposite directions by two pump lasers. Near the generation threshold, the laser exhibits temporal and spectral pulsations similar to those observed in $[14,15,18,19]$. However, as pump power increases well above the threshold, the generation becomes quasi-cw with a spectrum of only $\sim 1.5 \mathrm{~nm}$ linewidth localized at the Raman gain spectral maximum. The developed random DFB fiber laser constitutes a new type of laser different from both classical random lasers and conventional lasers. The spectrum localization and narrowing as well as its quasi-cw regime are explained by turbulentlike randomization of the phases and amplitudes of the generated spectral components via their multiple nonlinear (four-wave-mixing) interactions and dispersion in a long fiber. A different nonlinear mechanism of stabilization is proposed in [21]: Pump laser intensity fluctuations may induce phase fluctuations and the corresponding spectral broadening of the generated radiation via a cross-phase-modulation process, thus suppressing the SBS-induced instabilities.

In this article, we extend the study initiated in [20] and demonstrate $\mathrm{cw}$ narrow-spectrum generation based on a random distributed feedback in a number of cavity configurations with or without point reflectors in a nonsymmetric configuration with one pump laser. The temporal, optical, and rf spectra of the developed lasers are studied in detail. We compare the generation thresholds for different cavities calculated numerically and measured experimentally. The conventional cavity length limit and the minimal threshold pump power required for the generation based purely on a random distributed feedback are determined.

\section{LASER WITH CONVENTIONAL CAVITY}

To generate cw narrowband radiation we have used a standard telecom fiber span (SMF-28). The fiber was pumped through a wavelength-division multiplexer by a pump laser providing up to $2 \mathrm{~W}$ at center wavelength of $1455 \mathrm{~nm}$ (Fig. 1). A pump wave propagating through the fiber induces a distributed Raman amplification of the Stokes wave at longer wavelengths. The Raman amplification profile has a maxima near $1556 \mathrm{~nm}$ for the pump used [see Fig. 2(a)], and the gain coefficient is $g_{R}=0.39 \mathrm{~km}^{-1} \mathrm{~W}^{-1}$ in the spectral maximum. The fiber length $L=41 \mathrm{~km}$ is chosen in such a way that it is close to the amplification length roughly estimated
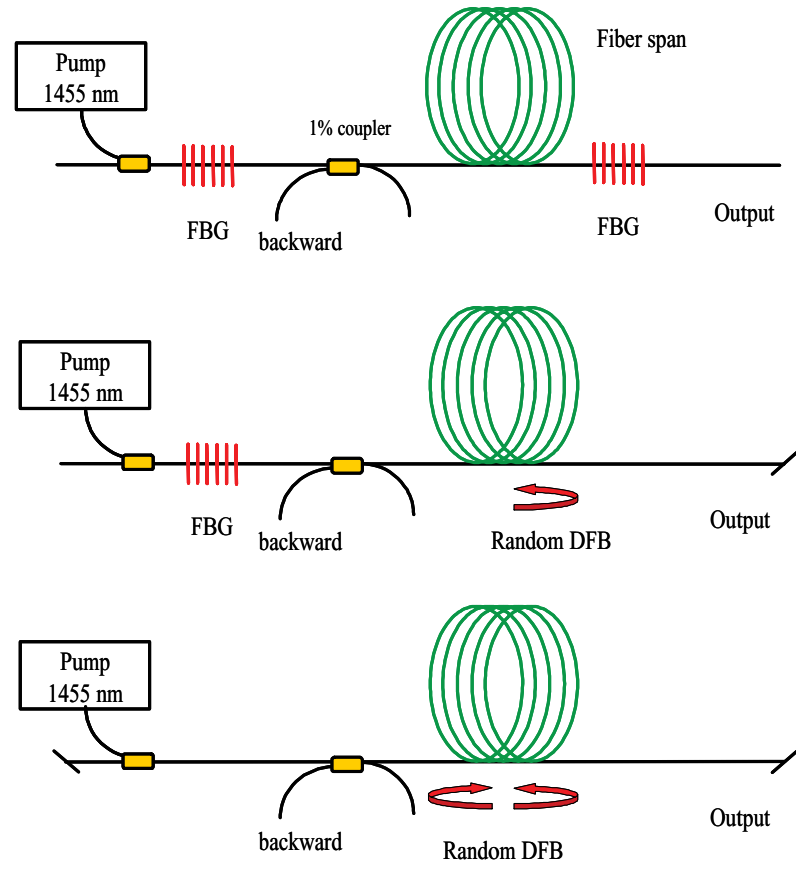

FIG. 1. (Color online) Raman fiber lasers (a) with a conventional linear cavity formed by two FBGs, (b) with a cavity formed by one FBG and a random distributed feedback, and (c) with a cavity formed only by a random distributed feedback.

through the gain and loss balance condition, $g_{R} P_{P}\left(L_{\mathrm{RS}}\right)=\alpha$, giving length $L_{\mathrm{RS}}=\ln \left(g_{R} P_{0} / \alpha\right) / \alpha_{P} \approx 30-40 \mathrm{~km}$ (depending on pump power $P_{0}$ ) required for the lasing based on the pure random distributed RS feedback (see [20] for the details). Here linear losses are $\alpha_{p}=0.25 \mathrm{~dB} / \mathrm{km}$ for the pump wave and $\alpha=0.197 \mathrm{~dB} / \mathrm{km}$ for the Stokes wave. This scheme differs from the configuration presented in [20] by the pumping arrangement - the fiber is pumped from one side using one pump laser only.

In this article, we investigate effects of the random distributed feedback starting from the conventional RFL scheme. It is well-known that if a Raman pumped fiber is placed inside the cavity formed by two FBGs of the same central wavelength [Fig. 1(a)], then an efficient and temporally and spectrally stable generation at the Stokes wavelength is easily achieved [22]. Depending on the Raman gain media and the cavity-forming FBGs, RFLs can be designed to operate at almost any wavelength in the near-infrared region [22]. The typical Stokes wave intracavity power, measured near the FBG, is shown in Fig. 2(b). The laser operates in a quasi-cw regime with a clear laser threshold and nearly linear growth of the Stokes power above the threshold, having a good generation efficiency of $\sim 30 \%$. The generation spectrum is centered at the FBG central frequency and has a specific trianglelike shape (in log scale) with exponential tails broadened due to turbulentlike mixing of different longitudinal modes [23] [Fig. 2(c)]. It is interesting to note that the powers of the forward (traveling from left to right) and backward (from right to left) Stokes waves are equal near the generation threshold, as it has to be for the high- $Q$ cavity. However, while pump power increases, the power of the forward (i.e., just reflected from the mirror) wave strongly saturates, thus indicating that the spectrum of 

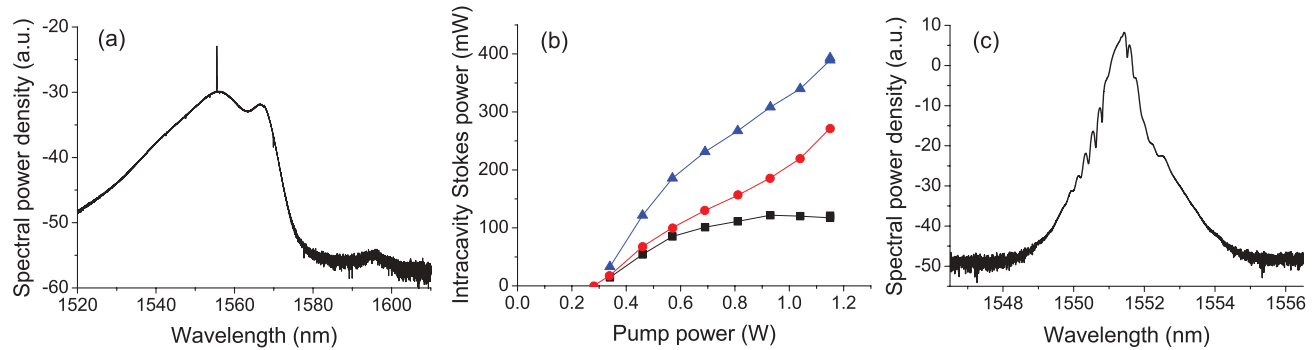

FIG. 2. (Color online) (a) A Raman gain profile of the fiber used. At the top of the profile, the laser generation starts. (b) A conventional RFL [Fig. 1(a)] intracavity power: forward (black boxes), backward (red circles), and Stokes waves powers together with the total intracavity Stokes wave power (blue triangles) versus pump power. (c) Backward Stokes wave spectrum measured just near the left FBG at a pump power of $1.2 \mathrm{~W}$.

the backward Stokes wave (i.e., of the wave incident on the mirror) becomes broader than the mirror reflection profile, having a bandwidth of $\sim 1 \mathrm{~nm}$ only.

It has been shown earlier that a clear mode structure with spacing $\Delta=c / 2 n L$ is observable in the rf spectra for all lengths up to $L=270 \mathrm{~km}$, for which $\Delta \sim 0.38 \mathrm{kHz}$ [13]. With increasing power the mode structure is washed out because of nonlinear turbulentlike dephasing that leads to broadening of the intermode beating peaks larger than the mode spacing [24]. For the studied configuration, the measured value is $\Delta \sim 2.4 \mathrm{kHz}$. The radiation is spatially well confined by the fiber waveguide, providing good beam quality of $M^{2}<1.2$.

As the fiber length in our case is chosen in such a way that the amplified Rayleigh backscattering is sufficiently high to impact the laser performance, the conventional RFL operation can be affected by random distributed feedback. Indeed, we have checked that further lengthening the cavity up to $165 \mathrm{~km}$ leads to instabilities in the optical spectra induced by the RS similar to those observed in $[17,18]$. The spiky dynamics in the spectra is more pronounced near the generation threshold. Further increase of the pump power results in stabilization of the generation.

\section{LASERS OPERATING VIA A RANDOM DISTRIBUTED FEEDBACK}

In the situation when one of the FBGs is removed [see Fig. 1(b)], the laser generation is possible only if the extremely weak distributed Rayleigh backscattering can provide a suffi- cient feedback to build up the laser cavity together with the FBG and satisfy the generation condition with the integral gain overcoming accumulated fiber losses over a cavity round trip. Indeed, it is demonstrated that such a scheme has a clear laser threshold. The output power grows linearly and reaches $120 \mathrm{~mW}$, having approximately $20 \%$ slope efficiency [Fig. 3(a)]. Note that we have ensured that the setup does not comprise any lumped feedback in the fiber: Fiber ends are angle cleaved or isolators were used, and splice losses are minimized. These precautions are important as it was observed that an insertion of only $4 \%$ Fresnel reflection on the fiber end was sufficient to change the laser performance: The threshold becomes lower $(0.5 \mathrm{~W})$, the slope efficiency is higher ( $\sim 2$ times), and longitudinal modes defined by the cavity length of $41 \mathrm{~km}$ are clearly resolved in the $\mathrm{rf}$ spectrum.

The laser with the cavity formed by one FBG and a random distributed feedback based on Rayleigh backscattering exhibits two different operation regimes similar to a pure random DFB laser [20]. Near the generation threshold stochastic pulses are generated in the Stokes wave [Fig. 4(a)], with the optical spectrum consisting of two components: a narrow peak associated with the FBG reflection spectrum, centered at $1551 \mathrm{~nm}$, and a broadband spiky continuum [see Fig. 3(b)]. The measured rf spectrum shows no indication of longitudinal modes beating, in contrast to the cavity with two FBGs or one FBG cavity assisted with $4 \%$ Fresnel reflection from the fiber facet. Instead, the rf spectrum (Fig. 4) clearly shows a presence of multiple SBS processes: Well-pronounced peaks near 11 and $22 \mathrm{GHz}$ coincide with the Brillouin shift. The pulsed
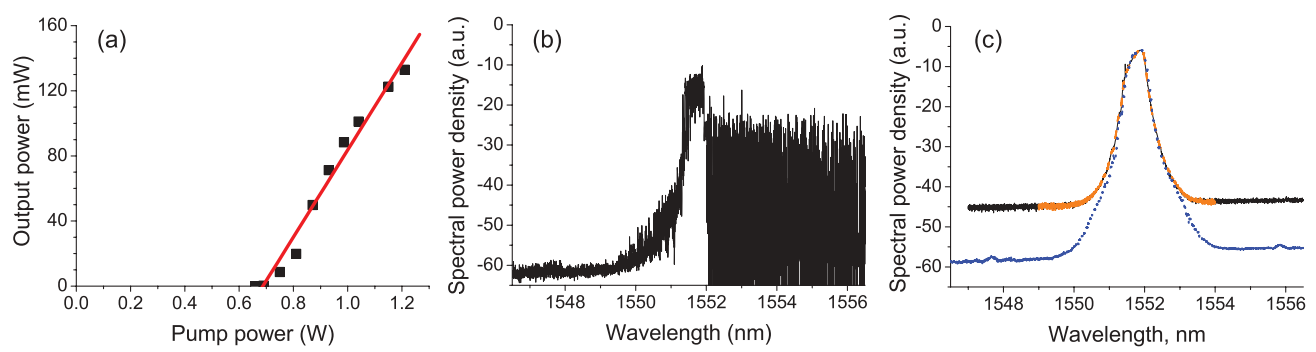

FIG. 3. (Color online) (a) Output power of the laser with the cavity formed by FBG and a random distributed feedback [Fig. 1(b)]. Output laser spectra measured at pump power level of $P=0.8 \mathrm{~W}$ (b) and $P=1 \mathrm{~W}$ (c) [blue dotted (lower) line]. Spectrum of the backward-propagating Stokes wave measured near the FBG for the fiber span length of $L=41 \mathrm{~km}$ (c, black line) and $L=165 \mathrm{~km}$ (c, orange dashed line, coinciding with black line) at the same pump power level $P=1 \mathrm{~W}$. All spectra are normalized to have equal amplitudes in their spectral maxima. 

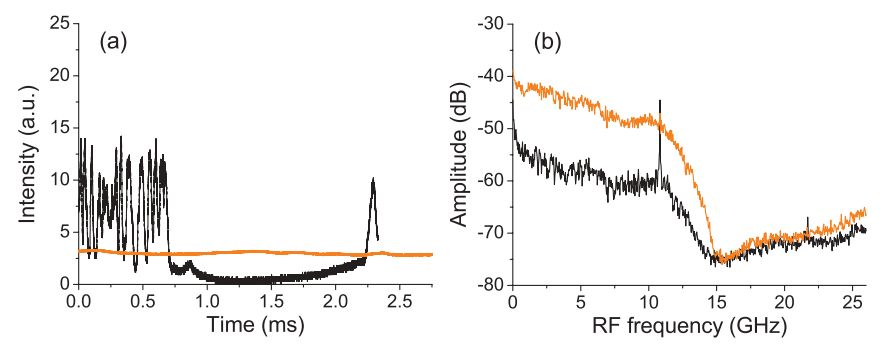

FIG. 4. (Color online) (a) Temporal behavior of the laser with the cavity formed by FBG and a random distributed feedback [Fig. 1(b)] in a pulsed $(P=1 \mathrm{~W}$, black line $)$ and quasistationary $[P=1.15 \mathrm{~W}$, orange (gray) line] regimes. (b) Typical rf spectra in pulsed ( $P=1 \mathrm{~W}$, black line) and quasistationary $[P=1.15 \mathrm{~W}$, orange (gray) line] regimes.

behavior of the random distributed feedback (RDFB) laser in this regime is similar to a self- $Q$-switched laser with combined RS-SBS feedback and has been studied extensively $[17,18]$. The laser provides stable TEM00-mode beam profile because of fiber waveguide properties.

As pump power increases above $0.8 \mathrm{~W}$, the situation is completely different: The laser goes to the quasi-cw regime [Fig. 4(a)], despite the random nature of the distributed feedback. At the same time, there is no evidence of SBS processes in the rf spectrum (Fig. 4). The optical spectrum becomes well-localized [see Fig. 3(c)], having full width at half maximum less than $0.5 \mathrm{~nm}$, which is comparable with the typical spectral width of the laser with conventional FBG-based cavity. The generated spectrum central frequency is defined by the FBG central frequency as in the laser with conventional cavity. The used fiber length used is 2 orders of magnitude longer than the typical nonlinear length at power levels of $\sim 1 \mathrm{~W}$. Thus, the generated spectrum should be strongly affected by nonlinear spectral broadening during propagation as in ultralong RFLs [24]. However, the normalized generation spectra at different fiber ends (output radiation spectrum at the right fiber end and spectra of backward-propagating waves measured near the FBG at the left fiber end) are identical, having some equilibrium shape which does not change during propagation [Fig. 3(c)]. The only difference is the Amplified Spontaneous Emission (ASE) suppression ratio: It is around $40 \mathrm{~dB}$ for the backward-propagating wave, and reaches $55 \mathrm{~dB}$ for the output radiation that is close to the ASE suppression ratio for the spectra generated in the conventional cavity [see Fig. 2(c)]. Note that the actual level of the ASE far from the laser line is almost the same for the forward- and backward-propagating waves. Moreover, if the fiber length $L<L_{\mathrm{RS}}$, the ASE levels are equal, $P_{\mathrm{ASE}}(L)=P_{\mathrm{ASE}}(0)=$ $P_{\mathrm{ASE}} \exp (g \bar{P} L-\alpha L)$, where $\bar{P}$ is an average over the fiber pump power. At the same time, laser power may be different, resulting in different suppression ratio.

Finally, if all FBGs are removed [Fig. 1(c)], there is only a Raman pumped fiber forming the laser cavity. It can start to lase if a double random distributed Rayleigh backscattering forms a sufficient feedback per round trip to fulfill the lasing condition. When the pump power increases, a clear generation threshold is found [Fig. 5(a)]. Below the threshold, the measured spectrum corresponds to amplified spontaneous emission with the typical broadband Raman gain curve. Just above the threshold, the laser operates in the pulsed regime similar to the previous case of the cavity with one FBG. However, the pulsations are observed in a wider range of pump powers. The optical spectrum consists of random intense spikes localized now near the Raman gain profile spectral maxima [Fig. 5(b)]. At the highest pump power available in the experiment, $1.75 \mathrm{~W}$, the output spectrum becomes almost stationary, having two separate lasing lines of spectral width at half maximum of $\sim 1.7$ and $\sim 0.8 \mathrm{~nm}$ for the left and right lines, correspondingly [Fig. 5(c)]. The quasi-cw generation on both lines is surely possible under the appropriate increased pump power. Introducing the $4 \%$ reflection from both fiber ends stabilizes the generation and makes the generation threshold lower by $0.2 \mathrm{~W}$ of pump power. The rf spectrum indicates the presence of cascaded SBS processes at low pump powers and no SBS line in the quasi-cw regime. Thus, a pure RDFB can form the cavity and provide the quasi-cw lasing with a spectrum localized near the Raman gain profile spectral maxima.

\section{GENERATION THRESHOLDS}

In order to verify and better understand our experimental findings, we have examined theoretically and calculated numerically the lasing thresholds for different considered configurations. It has been previously shown [25] that the operation of an ultralong RFL can be accurately described by the average power steady-state laser equations that take into account all important effects such as pump depletion, amplified spontaneous emission noise, and double RS. The average power equations that account for Rayleigh backscattering and

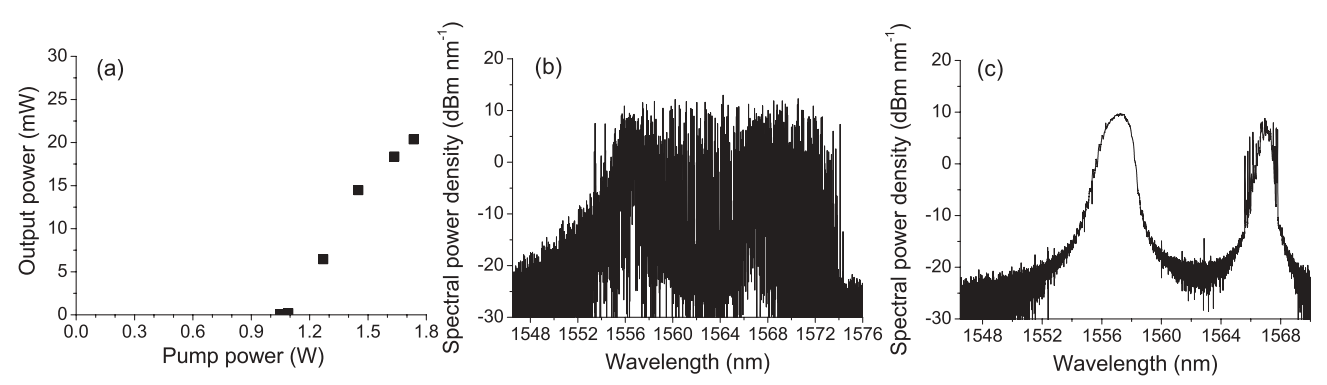

FIG. 5. (a) Output power of the laser with the cavity formed by a random distributed feedback only [Fig. 1(c)] versus the pump power. Output optical spectra measured at (b) pump power $1.45 \mathrm{~W}$ and (c) the highest available pump power, $1.75 \mathrm{~W}$. 
spontaneous emission read

$$
\begin{aligned}
\frac{d P_{p}^{ \pm}}{d z}= & \mp \alpha_{p} P_{p}^{ \pm} \mp g_{R} \frac{v_{p}}{v}\left[P^{+}+P^{-}+4 h v \Delta v\right. \\
& \left.\times\left(1+\frac{1}{\exp \left[h\left(v_{p}-v\right) / k_{B} T\right]-1}\right)\right] P_{p}^{ \pm} \pm \varepsilon_{p} P_{p}^{\mp}, \\
\frac{d P^{ \pm}}{d z}= & \mp \alpha P^{ \pm} \pm g_{R}\left(P_{p}^{+}+P_{p}^{-}\right)\left[P^{ \pm}+2 h v \Delta v\right. \\
& \left.\times\left(1+\frac{1}{\exp \left[h\left(v_{p}-v\right) / k_{B} T\right]-1}\right) \pm \varepsilon P^{\mp}\right],
\end{aligned}
$$

where + and - denote co- and counterpropagation of light in the fiber relative to the primary pump, respectively; $P_{p}$ and $P$ represent, respectively, the pump power at frequency $v_{p}$ and the power of the trapped first Stokes component at frequency $v ; \Delta v$ is the bandwidth of the generated radiation; and $\varepsilon_{p}=6 \times 10^{-5} \mathrm{~km}^{-1}$ is the RS coefficient for the pump wave. One can solve this equation set numerically using appropriate types of boundary conditions corresponding to the different types of the cavities considered, formed by point reflectors or random distributed feedback. The numerically calculated generation thresholds are presented in Fig. 6 for all experimentally realized cavities.

As expected, the generation threshold in the case of conventional cavity is increasing with the increased cavity length (black solid line in Fig. 6) as more pump is needed to overcome increased total losses in more lengthy cavity. More precisely, the generation threshold can be estimated as $P_{\text {th }}=\frac{\alpha \alpha_{p} L}{g_{R}\left[1-\exp \left(-2 \alpha_{p} L\right)\right]}$ (see [26]), that is, equal to $P_{\text {th }}=\frac{\alpha \alpha_{p} L}{g_{R}}$ for long fibers; that is, the threshold pump power is proportional to the fiber length $L$. However, while increasing the cavity length over $100 \mathrm{~km}$, the threshold value becomes to be lower than predicted by this simple consideration. This means that the feedback mechanism is gradually shifted from pure lumped feedback to random distributed feedback. Moreover, at $L_{\text {max }}>135 \mathrm{~km}$ the generation threshold power becomes independent of fiber length; thus, the a laser of such cavity length operates via random distributed feedback only.

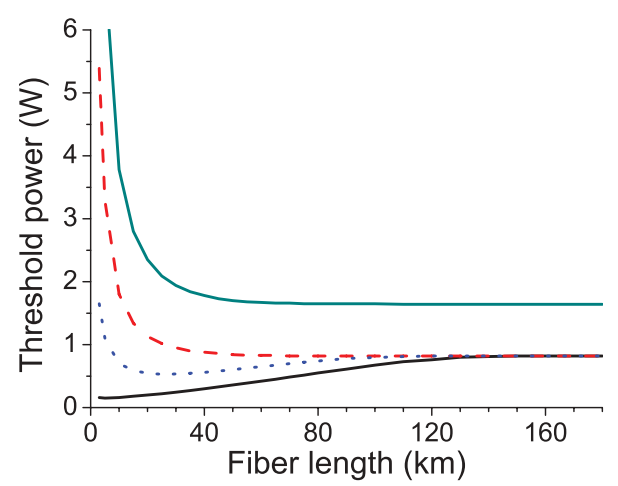

FIG. 6. (Color online) Numerically obtained lasing thresholds with single pump for the different types of the cavities: formed by two FBGs (black line), one FBG, a random distributed feedback with $4 \%$ reflection from other fiber end (blue dotted line) and without it (red dashed line), and random distributed feedback only [green (gray) line].
Neglecting the RS, the cavity formed by a single FBG and $4 \%$ fiber facet is just a conventional Fabry-Perot cavity. Laser generation in such a cavity is possible; however, the threshold should be higher compared to a cavity formed by two highly reflective FBGs, and the generation threshold should also increase with the cavity length. However, the situation is completely changed in the presence of the RS: The generation threshold is rapidly decreasing (blue dotted line in Fig. 6), thus indicating that even extremely weak random distributed RS plays a critical role providing sufficiently more intense feedback for the cavity than a $4 \%$ fiber facet. The longer the cavity, the more intense is the random feedback and the lower is the generation threshold. The minimal threshold is achieved at the optimal cavity length of $25 \mathrm{~km}$, which corresponds to the estimation for $L_{\mathrm{RS}}$ at the pump power $0.5 \mathrm{~W}, L_{\mathrm{RS}} \sim 25 \mathrm{~km}$. With further lengthening of the cavity, the fiber length becomes longer than the optimal amplification length increasing total losses and, therefore, the generation threshold increases again.

In the case of removal of the $4 \%$ fiber facet, the generation in such a cavity cannot be achieved without a random distributed RS feedback. When the cavity is short, the Rayleigh backscattering is negligible; therefore, the generation threshold is many times higher than in the case of conventional cavity (see red dashed line in Fig. 6). However, the longer the fiber, the more pronounced is the random distributed feedback; thus, the generation threshold rapidly decreases, reaching the almost constant value of $0.8 \mathrm{~W}$ at a fiber length $L_{\mathrm{RS}} \sim 40$ $\mathrm{km}$ calculated at pump power $0.8 \mathrm{~W}$. When the fiber length is increased further over this value, $L>L_{\mathrm{RS}}$, this should have no impact on generation performances of the laser, as the additional fiber does not provide an amplification, but only attenuation for the generated light. We have checked this prediction directly by comparing the generation thresholds and the generation spectra of two lasers: one of $41 \mathrm{~km}$ cavity and another one of $165 \mathrm{~km}$ cavity, both having only one FBG. We have found that generation thresholds, generated power measured near the FBG, and spectral shapes are identical for both lasers (see Fig. 3 to compare optical spectra).

Thresholds for all three cavity types converge toward a fixed value of around $0.8 \mathrm{~W}$ reached at a length of about $L_{\max } \sim 135 \mathrm{~km}$. Thus, for cavities longer than $L_{\max }$, there is no difference whichever cavity type is used: All of them generate via random distributed feedback only. Thus, none of them will have longitudinal mode structure at any pump power.

A cavity formed by a RDFB only has the highest generation threshold [green (gray) solid line in Fig. 6]. The fiber length needed for achieving the generation with lowest generation threshold seems to be the same for the pure RDFB- and single FBG-based cavities. This means that both distributed Rayleigh "mirrors" for single and double RS are not separated in coordinate and are formed in the same piece of fiber pumped by a single pump laser. Note, that the minimal generation threshold for pure RDFB type of cavity is exactly 2 times higher than that for the single FBG configuration.

It is interesting to compare the results of the present investigation with results of [20] where the symmetrical configuration of total length of $83 \mathrm{~km}$ pumped by two pump sources in opposite directions from the fiber center was discussed. The symmetrical configuration of [20] have 
two separated Rayleigh mirrors. The second Rayleigh mirror acts as a highly reflective mirror reflecting light back to the cavity. Thus, the configuration comprising 2-times-shorter fiber $(41 \mathrm{~km}$ instead of $83 \mathrm{~km})$, one pump from the fiber end, and one FBG [Fig. 1(b)] can be treated as a "half" of the symmetrical configuration of [20] in terms of cavity length, total pump power, and pump and Stokes power distributions along the fiber. Indeed, the predicted length limit for the symmetrical configuration, $L_{\max } \sim 270 \mathrm{~km}$ (see [20]) is two times larger than the $L_{\max }$ value of $\sim 135 \mathrm{~km}$ obtained here (see Fig. 6). The minimal generation threshold in a symmetrical configuration, $1.75 \mathrm{~W}$, is approximately 2 times higher than that in the half of the setup having $0.8-\mathrm{W}$ threshold. At the same time, for the pure RDFB configuration of Fig. 1(c) having also two distributed mirrors, the minimal generation threshold, $1.8 \mathrm{~W}$, is equal to that in the symmetrical cavity of double length [20], as it should be.

Finally, note that the theoretical analysis presented here does not take into account SBS processes. As a result, numerical simulations cannot describe the pulsed regime involving cooperative RS-SBS processes and, therefore, predict the generation thresholds of such operation that are lower than thresholds of the quasi-cw regime. Indeed, the cavity type of Fig. 1(b) has the experimentally measured threshold of pulsed operation of $0.7 \mathrm{~W}$ [see Fig. 3(a)], lower than the predicted threshold of $1 \mathrm{~W}$ for $\mathrm{cw}$ operation. However, the quasi-cw generation in this cavity type starts in experiments from the pump power of $1 \mathrm{~W}$ [see Fig. 3(c)], which is in good agreement with the numerically obtained value (Fig. 6, red dashed line). The same is true for the cavity type of Fig. 1(c), having pure RDFB only: The pulsed operation starts at powers as low as $1 \mathrm{~W}$ [Fig. 5(a)], while the almost quasi-cw generation is possible at $1.75 \mathrm{~W}$ of pump power [Fig. 5(c)], which is close to the theoretically predicted value of $1.8 \mathrm{~W}$ (see the green (gray) solid line in Fig. 6).

\section{CONCLUSION}

We have extended here our study of a new type of laserRDFB fiber lasers-which were demonstrated in [20]. We have described in detail the generation properties of such lasers, focusing on different cavity configurations: conventional cavity formed by two FBGs, cavity formed by one FBG, and a random distributed-feedback- and a pure random distributed-feedback-based cavity. Quasi-cw generation of a well-localized spectrum is obtained in all configurations.
In cavities comprising FBGs, the central frequency of the generated spectrum is defined by the reflector spectral profile, while in the pure random distributed-feedback cavities, the generation wavelength is defined solely by the positions of the Raman gain maxima. It is shown that RS limits the conventional cavity length with a single pump to a length of $135 \mathrm{~km}$ and that the minimal required pump power needed for the generation based on random distributed feedback can be as low as $0.8 \mathrm{~W}$. Overall, the obtained results clearly prove that the random distributed feedback based on RS plays a fundamental role in long fiber lasers.

Despite the experimental demonstration of random distributed feedback fiber lasers, many fundamental questions about their operation remain open. A full understanding of the mechanism of generation of stable quasi-cw radiation with a well-localized spectrum despite the random nature of the feedback is yet to come. There are different qualitative scenarios [20,21], but there is no quantitative description of the temporal and spectral properties of such lasers. Investigations of the spectral structure and phase and amplitude fluctuations of spectral components and their correlations can help define the degree of spatial coherence of the generated radiation and clarify mechanisms responsible for the stabilization. As the presented lasers display properties of both random and conventional lasers, it would be interesting to study a photon statistics which can be Bose-Einstein statistics as in noncoherent random lasers or the usual Poisson statistics as in conventional cavity-based lasers.

On the other hand, the proposed RDFB fiber lasers have great potential to be used in practical applications. An interesting direction of further study of RDFB fiber lasers is their tunable operation, which seems to be easily achieved by tuning the pump laser in FBG-free cavity configurations, or tuning FBGs in hybrid setups. Recently, a RDFB laser operating at two wavelengths simultaneously has been demonstrated [27], and the possibility of further increasing the number of wavelengths seems to be fully feasible. Finally, the general idea of the generation based on the random distributed feedback can be transferred to a shorter spectral band where the RS coefficient is higher.

\section{ACKNOWLEDGMENTS}

The authors acknowledge financial support of EPSRC EP/E015646/1 and Russian Ministry of Education and Science grants.
[1] H. Cao, J. Phys. A 38, 10497 (2005).

[2] D. S. Wiersma, Nat. Phys. 4, 359 (2008).

[3] V. S. Letokhov, Sov. Phys. JETP 26, 835 (1968).

[4] V. M. Markushev, V. F. Zolin, and Ch. M. Briskina, Zh. Prikl. Spektrosk. 45, 847 (1986).

[5] C. Gouedard, D. Husson, C. Sauteret, F. Auzel, and A. Migus, J. Opt. Soc. Am. B 10, 2358 (1993).

[6] N. M. Lawandy, R. M. Balachandran, A. S. L. Gomes, and E. Sauvain, Nature (London) 368, 436 (1994).

[7] J. Fallert et al., Nat. Photonics 3, 279 (2009).

[8] D. S. Wiersma, Nat. Photonics 3, 246 (2009).
[9] V. Milner and A. Z. Genack, Phys. Rev. Lett. 94, 073901 (2005).

[10] C. J. S. de Matos, L. de S. Menezes, A. M. Brito-Silva, M. A. Martinez Gamez, A. S. L. Gomes, and C. B. de Araujo, Phys. Rev. Lett. 99, 153903 (2007).

[11] J. Senior, Optical Fiber Communications: Principles and Practice (Prentice Hall, Upper Saddle River, NJ, 2008).

[12] G. P. Agrawal, Fiber-optic Communication Systems (Wiley Interscience, Malden, MA, 2002).

[13] S. K. Turitsyn, J. D. Ania-Castañón, S. A. Babin, V. Karalekas, P. Harper, D. Churkin, S. I. Kablukov, A. E. El-Taher, E. V. 
Podivilov, and V. K. Mezentsev, Phys. Rev. Lett. 103, 133901 (2009).

[14] C. Chen, H. Lee, and Y. Cheng, in Optical Fiber Communication Conference, Technical Digest (Optical Society of America, 2003), paper TuC2.

[15] F. Vanholsbeeck, S. Coen, P. Emplit, C. Martinelli, and T. Sylvestre, Opt. Lett. 29, 998 (2004).

[16] S. V. Chernikov, Y. Zhu, J. R. Taylor, and V. P. Gapontsev, Opt. Lett. 22, 298 (1997).

[17] A. A. Fotiadi and R. V. Kiyan, Opt. Lett. 23, 1805 (1998).

[18] G. Ravet, A. A. Fotiadi, M. Blondel, and P. Megret, Electr. Lett. 40, 528 (2004).

[19] O. Frazao, C. Correia, J. L. Santos, and J. M. Baptista, Meas. Sci. Technol. 20, 045203 (2009).

[20] S. K. Turitsyn, S. A. Babin, A. E. El-Taher, P. Harper, D. V. Churkin, S. I. Kablukov, J. D. Ania-Castañón, V. Karalekas, and E. V. Podivilov, Nat. Photonics 4, 231 (2010).
[21] A. A. Fotiadi, Nat. Photonics 4, 204 (2010).

[22] E. M. Dianov, I. A. Bufetov, M. M. Bubnov, M. V. Grekov, S. A. Vasiliev, and O. I. Medvedkov, Opt. Lett. 25, 402 (2000).

[23] S. A. Babin, D. V. Churkin, A. E. Ismagulov, S. I. Kablukov, and E. V. Podivilov, J. Opt. Soc. Am. B 24, 1729 (2007).

[24] S. A. Babin, V. Karalekas, E. V. Podivilov, V. K. Mezentsev, P. Harper, J. D. Ania-Castañón, and S. K. Turitsyn, Phys. Rev. A 77, 033803 (2008).

[25] J. D. Ania-Castañón, T. J. Ellingham, R. Ibbotson, X. Chen, L. Zhang, and S. K. Turitsyn, Phys. Rev. Lett. 96, 023902 (2006).

[26] S. A. Babin, D. V. Churkin, and E. V. Podivilov, Opt. Commun. 226, 329 (2003).

[27] A. E. El-Taher, M. Alcon-Camas, S. A. Babin, P. Harper, J. D. Ania-Castañón, and S. K. Turitsyn, Opt. Lett. 35, 1100 (2010). 